\title{
WCC 2016-106: Deep Vein Thrombosis: Presentation and Clinical Risk Factors Among the Patients at a Tertiary Care Hospital \\ Bhupal Sri Ram Vallabhaneni
}

\section{Back ground:}

Deep vein thrombosis (DVT) is a serious and potentially fatal disease that can complicate and lead to catastrophe like pulmonary embolism and death. Number of clinical risk factors predisposes the patient to its development, risk of DVT increases in proportion to the number of clinical risk factors present in a case. Not much is known about the prevalence of the clinical risk factors of DVT among the patients in our part of the country. This study was carried out to determine frequency of the clinical risk factors in patients clinically suspected of DVT at Nizam's Institute of Medical Sciences, Hyderabad.

\section{Methods:}

This Cross sectional was conducted at Department of Cardiology, Nizam's Institute of Medical Sciences, Hyderabad during period of two years, from November 2013 to November, 2015. All consecutive patients suspected of Deep Vein Thrombosis (DVT) were enrolled. DVT was diagnosed on Duplex ultrasonography. A separate proforma was filled for each case entering in to the study for data collection. Statistics were performed for data presentation.

\section{Results:}

Total 61 cases were enrolled, out these 61 cases 19 (31.14\%) were male and 42 (68.86\%) female. Mean age of the male was 52.21 years \pm 18.99 , and female cases had 34.67 years $\pm 8.9(p=0.000)$. Of the 61 cases under the study DVT was detected in 48 (78.68\%) cases; of these 48 cases 14 (29.16\%) were male and 34 (70.83\%) female.

\section{Conclusion:}

Immobility was the most frequent risk factor in our cases.

Bhupal Sri Ram Vallabhaneni,

DM Student, Department Of Cardiology, NIMS, Hyderabad, India,.

Corresponding Author: Bhupal Sri Ram Vallabhaneni,

Email:vsrbhupal@yahoo.com 